\title{
Changes in platelet functional parameters and CD62 P expression in liver cirrhosis
}

\author{
Xianghong G, Guanping C, Fenghua Y, *Jiayin W \\ Center Department of Clinical Laboratory Medicine, Linyi People’s Hospital, Linyi 276003, China
}

\begin{abstract}
Background: Hepatic impairment, portal hypertension, and multi-systemic damage could occur during liver cirrhosis's late stage. Bleeding is a complication of hepatic cirrhosis along with several changes including blood platelet count (BPC), mean platelet volume (MPV), platelet crit (PCT) and expression of platelet CD62P. Blood platelet count (BPC), mean platelet volume (MPV), platelet distribution width, and other indices are indirect reflections of CD62P parameters.

Objective: To investigate the changes in platelet functional parameters and CD62 P expression in liver cirrhosis as a possible guide in clinical treatments and prognoses of liver cirrhosis.

Methods: CD62P was tested by flow cytometry in liver cirrhosis. BPC, MPV, and PCT in peripheral blood were tested using an auto blood cell analyzer. Data were analyzed using SPSS11.0.

Results: The values of CD62P and MPV in patients was significantly higher than those of healthy donors $(\mathrm{P}<0.01)$, while the values of BPC and PCT were significantly lower than those of the control group $(\mathrm{P}<0.01)$

Conclusions: CD62P, BPC, MPV, and platelet crit (PCT) show several changes in liver cirrhosis. It is useful to understand the relationship between hepatic cirrhosis severity and CD62P, BPC, MPV, PCT, timely monitoring of CD62P for treatment of hepatic cirrhosis in clinical treatment and prognosis.

Keywords: Liver cirrhosis, granula protein, platelet parameters, expression

African Health Sciences 2013; 13(4): 1079 - 1083 http://dx.doi.org/10.4314/ahs.v13i4.31
\end{abstract}

\section{Introduction}

Cirrhosis is the final stage attained by various chronic hepatic diseases after years or decades of slow progression. It can thus be prevented by appropriate screening for chronic liver diseases so that they can be treated in time. Chronic liver diseases do not usually cause any symptoms. However, hepatic impairment, portal hypertension, and multi-systemic damage could occur during the late stage of liver cirrhosis. Moreover, hypersplenism ${ }^{1,2}$, thrombocytopenia ${ }^{3-6}$, and consumption of blood coagulation factors and antibodies directed against platelets ${ }^{7-9}$ could lead to bleeding tendency. Granula protein on the platelet surface (CD62P) has very high sensitivity and specificity to platelet activation; thus, it is regarded as a special index of platelet activation ${ }^{10-}$ ${ }^{12}$. Platelets are formed by fragments of macrophage kytoplasm, and interact with mono-macrophages in the spleen. The mean platelet volume (MPV) reflects the active compounds and granula in the platelet.

\begin{tabular}{|l|}
\hline *Corresponding author: \\
Jiayin Wang \\
Center Department of Clinical Laboratory \\
Medicine \\
Linyi People's Hospital \\
No.27 Jiefang Road \\
Linyi276003, P.R. China \\
Email: xxb2001@yeah.net \\
\hline
\end{tabular}

African Health Sciences Vol 13 Issue 4 December 2013
The larger the size of the platelet, the more glycogen, adenine, nucleotide, and normal phosphates it contains and the higher its active functions become. Smaller-sized platelets have lower functions. Blood platelet count (BPC), MPV, and other indices are indirect reflections of its parameters ${ }^{13}$. Reports show that BPC, MPV, and platelet crit (PCT) exhibit some changes in liver cirrhosis. Decreased MPV may be related to the presence of endotoxins, as well as to increased immunoglobulin, both having the ability to induce sustained platelet activation, thereby leading to the release of active compounds that cause granula exhaustion, platelet shrinkage, and decreased reservation ${ }^{14}$.

In this study, CD62P was tested by flow cytometry in liver cirrhosis. BPC, MPV, and PCT in peripheral blood were tested using an auto blood cell analyzer. Understanding the relationship between hepatic cirrhosis severity and CD62P, BPC, MPV, PCT, timely monitoring of CD62P, and other platelet parameters is highly essential in preventing hemorrhage and coagulation disorders during the early stage of hepatic cirrhosis and may serve as a guide in clinical treatments and prognoses. 


\section{Methods}

\section{Patients}

Up to 32 cases of hepatic cirrhosis were studied, among whom were 25 male and 7 female. Their age range was $35-68$ years, and the average age was $51 \pm 15$. The diagnostic criteria were in accordance with the 5th National Infectious Diseases and Parasite Conference, and created from combined clinical manifestations, liver function, ultrasound, and CT scan findings. The patients were classified according to by Child-Pugh (REF) as follows: Grade A, 12 cases; Grade B, 10 cases; and Grade C, 10 cases, which was used for assessing the prognosis of cirrhosis. The higher score, the worse of liver reserve function (Grade A, 5-6, the risk of operation is low; Grade B, 7-9, the risk of operation is higher than Grade A; Grade C, e"10, the risk of operation is the highest and the prognosis is the worst). All the patients had no heart, brain, kidney, or diabetic problems; they were also not suffering from acute infections. Six cases were confirmed to have upper gastrointestinal tract bleeding through gastroscope examination and a positive occult blood (OB) test.

The control group comprising 20 cases of normal patients underwent a health checkup. Among the 20 cases, 10 were male and 10 were female, aged 21 to 50 years, with an average age of $38 \pm 12$. All cases in the patient group and in the control group did not take any anticoagulant one week before the test. This study was conducted in accordance with the declaration of Helsinki. This study was conducted with approval from the Ethics Committee of Linyi People's Hospital. Written informed consent was obtained from all participants.

\section{Detection of CD62P}

Approximately $2 \mathrm{ml}$ of fasting blood samples from both patient and control groups were collected with $3.84 \%$ citric acid sodium. Then the samples were placed in $1 \%$ paraformaldehyde at $4{ }^{\circ} \mathrm{C}$ for $15 \mathrm{~min}$. The samples underwent centrifugation at $800 \mathrm{r} / \mathrm{min}$ for $5 \mathrm{~min}$. The platelet-rich supernatant was then separated. Up to $50 \mu \mathrm{l}$ supernatant was mixed with CD62P monoclonal antibody (Becton-Dickinson) labeled with phycoerythrin, 20 ul aliquot was mixed and used for testing, and another $50 \mu$ platelet-rich plasma was placed in a tube and mixed with phosphate buffer solution (PBS) for use as control. Both tubes were reacted for $20 \mathrm{~min}$ away from light, and then mixed with $1 \mathrm{ml}$ of $1 \%$ paraformaldehyde at $4{ }^{\circ} \mathrm{C}$. The tubes were poached with PBS and underwent centrifugation at $2000 \mathrm{r} / \mathrm{min}$ for $5 \mathrm{~min}$. The supernatant was withdrawn and the procedure was repeated twice, adjusting the platelet concentration to $10 \times 10^{\circ} / \mathrm{L}$, preserving at $4^{\circ} \mathrm{C}$, and testing with flow cytometry assay (Becton-Dickinson) within 24 hours. All data were analyzed using Cell Quest Plot software. Platelet CD62P positive rate was obtained as a result.

\section{Detection of BPC, MPV, and PCT}

Up to $2 \mathrm{ml}$ samples with ethylenediaminetetraacetic acid (EDTA) anticoagulant and special anticoagulant $\left(1.5 \mathrm{mg} / \mathrm{ml}\right.$ to $2.2 \mathrm{mg} / \mathrm{ml}$ EDTA- $\left.\mathrm{K}_{2}\right)$ were taken from both patient and control groups. Subsequently, they were tested using an M-533 auto blood cell analyzer. The process lasted for approximately 2 hours. Three kinds of whole blood were used as control. When all the results were within the normal range, samples were taken.

\section{Statistical analysis}

Data were expressed using mean \pm standard deviation $(\bar{x} \pm$ s). T-test was applied to obtain the mean of subgroup comparison in both groups. The data in the other group were tested with ANOVA. All data gathered were analyzed with SPSS11.0. $P<0.05$ was considered statistically significant.

\section{Results \\ Comparison of plasma CD62P, BPC, MPV, and PCT}

The testing results of CD62P, BPC, MPV, and PCT in the 32 cases of hepatic cirrhosis and in the 20 cases from the control group are shown in Table 1. CD62P and MPV in the cirrhosis group were higher than those in the control group $(P<0.05)$, whereas BPC and PCT were lower in the control group $(P<0.05)$. The differences are presented in table 1.

Table 1: Result of CD62P, BPC, MPV, and PCT in cirrhosis group and control group (X士s)

\begin{tabular}{llllll}
\hline Groups & Case & CD $62 \mathrm{P}(\%)$ & BPC $\left(10^{9} / \mathrm{L}\right)$ & MPV (fl) & PCT $(\%)$ \\
\hline Cirrhosis & 32 & $11.84 \pm 7.82^{*}$ & $43.96 \pm 21.69^{*}$ & $13.26 \pm 0.41^{*}$ & $1.06 \pm 0.72^{*}$ \\
Control & 20 & $2.74 \pm 1.05$ & $95.14 \pm 21.75$ & $9.73 \pm 0.80$ & $1.98 \pm 0.35$ \\
\hline
\end{tabular}

Data were expressed using average \pm standard deviation $(\bar{x} \pm s)$. T-test was applied to obtain the average of subgroup comparison in both groups. $P<0.05$ was considered statistically significant.

*Compared with control group, $P<0.05$. 
Analysis of CD62P, BPC, MPV, and PCT in liver cirrhosis

The testing value of platelet surface CD62P in Grade A cirrhosis patients was lower than that in Grade B $(P<0.05)$. BPC, MPV, and PCT showed no difference in both grades. The testing value of platelet surface CD62P in Grade C liver cirrhosis patients was higher than that in Grades A and B $(P<0.05)$. No marked difference was observed in various grades $(P>0.05)$. The results of CD62P, BPC, MPV, and PCT in different grades are shown in table 2.

Analysis of CD62P, BPC, MPV, and PCT in bleeding or non-bleeding group

The testing value of platelet surface CD62P (24.79 \pm 6.55$)$ was markedly higher than that in the no-bleeding group $(8.85 \pm 4.21)(\mathrm{P}<0.01)$, whereas BPC, MPV, and PCT exhibited no significant difference. The testing values of CD62P, BPC, MPV, and PCT in the upper digestive bleeding with hepatic cirrhosis group and in the non-bleeding group are shown in table 3. mediates the interaction between platelets and other cells, platelets aggregation and adherence by improving the interaction of cells. Platelets originate from bone marrow megakaryocytes and the number of megakaryocytes indicates the function of hematogenesis in the bone marrow. The testing value of BPC reflects the generation and decay of platelets. MPV reflects the metabolization of bone marrow megakaryocytes. PCT is a parameter reflecting the condition of granula and active substances in platelets. Thus the detection of BPC, MPV and PCT in peripheral blood is used for treatment of liver cirrhosis clinically ${ }^{16-17}$ since bleeding is a complication of liver cirrhosis.

This study showed that CD62P in liver cirrhosis patients was higher than that in the control group, whereas the number of platelets were lower in the control group. This finding indicates that the increase in CD62P was related to the overdestruction and activation of platelets in vivo. Therefore, CD62P in

Table 2: Testing result CD62P, BPC, MPV, PCT of platelet surface $(X \pm s)$

\begin{tabular}{llllll}
\hline Group & Case & CD62P (\%) & BPC (10 $/ \mathbf{L})$ & MPV (f1) & PCT (\%) \\
\hline Grade A & 12 & $6.68 \pm 2.31$ & $52.00 \pm 21.67$ & $10.51 \pm 0.39$ & $1.32 \pm 0.51$ \\
Grade B & 10 & $9.20 \pm 4.06^{*}$ & $40.50 \pm 22.14$ & $11.76 \pm 0.52$ & $1.24 \pm 0.76$ \\
Grade C & 10 & $20.67 \pm 7.61 * *$ & $37.50 \pm 17.54$ & $13.26 \pm 0.41$ & $1.06 \pm 0.72$ \\
\hline
\end{tabular}

Data were expressed using average \pm standard deviation $(X \pm s)$. T-test was applied to obtain the average of subgroup comparison in both groups. $P<0.05$ was considered statistically significant.

Grade B compared with Grade A, P $<0.05$; Grade C compared with Grade A and B, $P<0.05$

Table 3: Result of CD62P, BPC, MPV, and PCT on surface of platelet in upper digestive bleeding and non-bleeding group with hepatic cirrhosis $( \pm s)$

\begin{tabular}{llllll}
\hline Group & case & CD62P $(\%)$ & BPC $\left(10^{9} / \mathrm{L}\right)$ & MPV(fl) & PCT $(\%)$ \\
\hline Bleeding & 6 & $24.79 \pm 6.55^{*}$ & $35.67 \pm 27.80$ & $11.20 \pm 1.20$ & $1.05 \pm 0.71$ \\
Non-bleeding & 26 & $8.85 \pm 4.21$ & $46.58 \pm 21.90$ & $10.60 \pm 0.80$ & $1.31 \pm 0.50$ \\
\hline
\end{tabular}

Data were expressed using average \pm standard deviation $(\bar{x} \pm s)$. T-test was applied to obtain the average of subgroup comparison in both groups. $P<0.05$ was considered statistically significant.

*Comparison between bleeding group and non-bleeding group, $P<0.05$.

\section{Discussion}

CD62P is a surface maker for platelet activation, which is present in the granules of platelets. CD62P is a member of selectin family. It is expressed by activated platelets, megakaryocytes, and vascular endothelial cells. It is one of the important membrane proteins, which serve for coagulation ${ }^{15}$. CD62P hepatic cirrhosis patients was higher than that in the control group. CD62P was also related to the ChildPugh classification, indicating that the platelets were highly activated. To some extent, the destruction of platelets along with the severity of liver cirrhosis increased the value of $\mathrm{CD} 62 \mathrm{P}$ and destroyed more 
platelets. Meanwhile, CD62P was higher in the group with liver cirrhosis combined with upper GI bleeding than in the non-bleeding group, indicating the relation of platelet activation and liver cirrhosis severity. Therefore, testing the value of CD62P revealed the severity of cirrhosis. The number of platelets in various groups did not exhibit marked difference, indicating that this factor was not related to upper digestive bleeding. However, the number of platelets was related to platelet function. Thus, $\mathrm{CD} 62 \mathrm{P}$ is a good index that can be used to determine the activation and function of platelets.

This study found that the number of platelets and PCT was lower in liver cirrhosis patients, but their MPV was increased. Thompson et al. ${ }^{18}$ postulated that large-volume platelets contain more body density, higher activity, more rapid metabolism, more powerful adhesive capacity, and more bleeding. They found that MPV and CD62P were higher in liver cirrhosis patients than in the control group, whereas BPC and PCT were lower in hepatic cirrhosis patients than in the control group, indicating the decrease in the number of platelets and the change in quantity. The increase in CD62P and MPV could cause hypersplenism and destroy platelets ${ }^{19,20}$. The mononuclear macrophage system compensated for the excretion of large-volume platelets, whereas the platelets themselves exhibited morphology changes, such as giant cells and abnormities, possibly meeting the body's coagulate function requirement.

\section{Conclusion}

Dynamic monitoring of the expression of CD62P and the parameters of platelets is helpful in ascertaining the activities and function status of platelets. It is also useful in discovering early bleeding and coagulation disorders caused by hepatic cirrhosis, thus leading to early and accurate treatment and prognosis. Furthermore, results obtained from the dynamic monitoring of the expression of CD62P and the parameters of platelets can serve as guidelines for clinical treatments and prognosis. .

\section{References}

1. Ohira M, Ishifuro M, Ide K, et al. Significant correlation between spleen volume and thrombocytopenia in liver transplant patients: a concept for predicting persistent thrombocytopenia. Liver Transpl 2009; 15 (2): 208-215.

2. Akyüz F, Yekeler E, Kaymakoðlu S, et al. The role of thrombopoietin and spleen volume in thrombocytopenia of patients with noncirrhotic and cirrhotic portal hypertension. Turk $J$ Gastroenterol 2007; 18 (2): 95-99.

3. Nomura T, Kubota Y, Kitanaka A, et al. Immature platelet faction measurement in patients with chronic liver disease: a convenient marker for evaluating cirrhotic change. Int $\mathrm{L}$ ab Hematol 2010; 32 (3): 299-306.

4. Afdhal N, McHutchison J, Brown R, et al. Thrombocytopenia associated with chronic liver disease. J Hepatol 2008; 48 (6): 1000-1007.

5. Yao FY, Bass NM. Lamivudine treatment in patients with severely decompensated cirrhosis due to replicating hepatitis B infection.J Hepatol 2000; 33 (2): 301-307.

6. Rios R, Sangro B, Herrero I, Quiroga J, Prieto $J$. The role of thrombopoietin in the thrombocytopenia of patients with liver cirrhosis. Am J Gastroenterol 2005; 100 (6): 1311 1316.

7. Panasiuk A. Autoantibodies in chronic liver diseases. Rocz. Akad Med Bialymst 2001; 46: 106112.

8. Ho CH, Hou MC, Lin HC, Lee FY, Wu JC, Lee SD. Hemostatic changes in patients with liver cirrhosis. Zhonghua Yi Xue Za Zhi (Taipei) 1999; 62 (6): 376-382.

9. Pihusch R, Rank A, Göhring P, Pihusch M, Hiller E, Beuers U. Platelet function rather than plasmatic coagulation explains hypercoagulable state in cholestatic liver disease. J Hepatol 2002; 37 (5): 548-555.

10. van Kooten F, Ciabattoni G, Koudstaal PJ, Dippel DW, Patrono C. Increased platelet activation in the chronic phase after cerebral ischemia and intracerebral hemorrhage. Stroke 1999; 30 (3): 546-549.

11. Sedlmayr P, Grosshaupt B, Muntean W. Flow cytometric detection of intracellular platelet antigens. Cytometry 1996; 23 (4): 284-289.

12. Escolar G, White JG. Changes in glycoprotein expression after platelet activation: differences between in vitro and in vivo studies. Thromb Haemost 2000; 83 (3): 371-386.

13. Thompson CB, Jakubowski JA, Quinn PG, Deykin D, Valeri CR. Platelet size as a determinant of platelet function. J Lab Clin Med 1983; 101 (2): 205-213.

14. Laffi G, Mann F, Geesele P, et al. Evidence for a storage pool defect in platelets from cirrhotic

African Health Sciences Vol 13 Issue 4 December 2013 
patients with defective aggregation. Gastroenterology 1992; 103 (2): 641-646.

15. Xilin OuYang, Jinghan and Liu, Dayong Gao. Flow cytometry detection of CD $62 p$ reexpression by preserved platelets and it's value in evaluation of the quality of preserved platelets. Chinese Journal of Blood Transfusion. 2002, 15(2):7781.

16. Kai Li, Qiang Ou, Xuefeng Li, Minghuan Zhou and Jian Zhou. Clinical Significance on Detecting Parameters of Platelet in Liver Cirrhosis. Journal of Clinical Research. 2007, 24(1):51-52.

17. Miao Bao and Xiansheng Li. The analysis for testing results of platelet parameter and blood coagulation factor in hepatic cirrhosis. Shandong Medical Journal. 2009, 49(25):46-47.
18. Thompson CB, Eaton KA, Princiotta SM, Rushin CA, Valeri CR. Size dependent platelet subpopulations: relationship of platelet volume to ultrastructure, enzymatic activity, and function. BrJ Haematol 1982; 50 (3): 509-519.

19. Vardareli E, Saricam T, Demirustu C, Gulbas Z. Soluble P selectin levels in chronic liver disease: relationship to disease severity. Hepatogastroenterology 2007; 54 (74): 466-469.

20. Sanjo A, Satoi J, Ohnishi A, Maruno J, Fukata M, Suzuki N. Role of elevated platelet-associated immunoglobulin $G$ and hypersplenism in thrombocytopenia of chronic liver diseases. $J$ Gastroenterol Hepatol 2003; 18 (6): 638-644. 